\title{
Hyperglycemic Hyperosmolar State
}

\author{
Sanjeevani R Zadkar
}

\begin{abstract}
With increasing incidence of diabetes mellitus, complications associated with it are also increasing. The hyperglycemic hyperosmolar state $(\mathrm{HHS})$ is one of the common complications seen in old diabetics where patients have markedly increased serum glucose concentrations and hyperosmolality in absence of significant ketosis. Most common precipitating factor is infection. In HHS, levels of insulin in circulation reduce significantly while counterregulatory or stress hormones such as cortisol, catecholamines, glucagon, and growth hormone increase along with greater degree of dehydration. Hence, these patients, in addition to typical features of diabetes, generally present with dehydration and progressive mental deterioration. Basic hematological, biochemical, and radiological investigations are important to find and rule out precipitating factors and complications. Aggressive correction of the fluid and electrolyte imbalance along with the maintaining levels of insulin are very important components of the treatment. Simultaneously, identification and treatment of any underlying precipitating events should be done. Awareness, early diagnosis, and aggressive proper treatment play very crucial role in preventing the morbidity and mortality associated with HHS, which is 10 times more than diabetic ketoacidosis.

Keywords: Hyperglycemia, Hyperglycemic hyperosmolar state, Hyperosmolality.

Research and Innovation in Anesthesia (2019): 10.5005/jp-journals-10049-0076
\end{abstract}

\section{INTRODUCTION}

Dysglycemia is a common feature of critically ill patients. ${ }^{1}$ Incidence of diabetes is increasing exponentially in the past few decades. Management of dysglycemia has become an integral part of the critical care skill set. Diabetic ketoacidosis (DKA), hyperglycemic hyperosmolar state (HHS), and hypoglycemia are the commonest emergencies in patients with diabetes. Diabetic ketoacidosis is more frequently seen in young people with type 1 diabetes. ${ }^{2}$ The prevalence of ketone-prone type 2 diabetes mellitus (T2DM) syndrome is also increasing all over the world. ${ }^{1}$

August W von Stosch first described in 1828 diabetic coma. ${ }^{3}$ This condition was later termed as the hyperosmolar hyperglycemic state. The HHS is more common in older patients. Most commonly, it is associated with a precipitating event. ${ }^{4}$ Even though it is uncommon in children, it is reported that African American adolescents with type 2 diabetes have higher incidence. ${ }^{5}$ Obesity and increased consumption of carbohydrate-rich beverages have increased the incidence of HHS in pediatric patients. ${ }^{6}$ In 1980 , HHS incidence was 1 in 10,000; it climbed up by 2000 to reach 5 in $10,000{ }^{7}$

Early diagnosis is very crucial for HHS patients as the mortality associated with HHS is 10 times more than DKA. ${ }^{8}$

\section{Precipitating Factors}

Patients with well-controlled diabetes mellitus have minimal chance of developing HHS. ${ }^{9}$ Most common precipitating factor is infection. ${ }^{2}$ Urinary tract infection and pneumonia are the commonest among them. ${ }^{9}$ Critical events like cerebrovascular accident or myocardial infarction can stimulate the release of counterregulatory hormones and precipitate HHS. ${ }^{10,11}$ Certain drugs like glucocorticoid, thiazide diuretics, phenytoin, and beta blockers can precipitate the development of HHS. ${ }^{7}$

\section{Signs AND Symptoms}

Most of the patients presenting with HHS have history of polyuria, polydipsia, weakness, blurred vision, and progressive deterioration
Department of Anesthesiology, KEM Hospital, Parel, Mumbai, Maharashtra, India

Corresponding Author: Sanjeevani R Zadkar, Department of Anesthesiology, KEM Hospital, Parel, Mumbai, Maharashtra, India, Phone: +91 9167151512, e-mail: zsanju1@gmail.com

How to cite this article: Zadkar SR. Hyperglycemic Hyperosmolar State. Res Inno in Anesth 2019;4(2):32-35.

Source of support: Nil

Conflict of interest: None

in the mental status. ${ }^{2,12}$ Most of the patients with HHS have age more than 60 years with history of a precipitating factor and delay in receiving medical help. On examination, these patients commonly have signs of dehydration like dry mucous membranes, poor skin turgor, or hypotension similar to DKA. ${ }^{12,13}$

\section{Diagnosis}

The American Diabetes Association (ADA) has defined the diagnostic criteria for HHS, which include marked hyperglycemia blood glucose $>600 \mathrm{mg} / \mathrm{dL}$, minimal acidosis (venous $\mathrm{pH}>7.25$ or arterial $\mathrm{pH}>7.3$ and serum bicarbonate $>18 \mathrm{mEq} / \mathrm{L}$ ), absent to mild ketosis, and marked elevation in serum osmolality (effective osmolality $>320 \mathrm{mOsm} / \mathrm{L}) .{ }^{12}$ The HHS patients have very high levels of serum glucose and hyperosmolality with minimal or absent ketones. $^{7}$

As per the United Kingdom (UK) guidelines for diagnosis of $\mathrm{HHS}$, hyperglycemia is defined as $>540 \mathrm{mg} / \mathrm{dL}$ and the bicarbonate level should be greater than $15 \mathrm{mEq} / \mathrm{L}$. The other parameters are similar. The differences in the diagnostic criteria are minimal. The most important are in calculation of osmolality and assessment of severity. ${ }^{14}$

\section{Pathophysiology}

Pathophysiology of DKA and HHS has many common components except the insulin deficiency in HHS is relative. ${ }^{1}$ In HHS, sufficient amount of insulin is available to prevent lipolysis. The amount of 
insulin required to prevent lipolysis is one-tenth of the insulin levels required for glucose uptake. In HHS, levels of insulin in circulation reduce significantly while counterregulatory or stress hormones such as cortisol, catecholamines, glucagon, and growth hormone increase along with greater degree of dehydration. ${ }^{10,15}$ Dehydration in HHS is of greater severity compared to DKA. Mixed mild ketotic hyperosmolar conditions can coexist. Nearly $33 \%$ of DKA patients have components of HHS. ${ }^{16,17}$ Increased serum osmolarity can have profound effect on the brain. To maintain the intracellular volume, the brain produces idiogenic osmoles. Idiogenic osmoles are osmotically active substances. The effect of the production of osmoles is to prevent fluid movement from the intracellular space into extracellular space. This helps in maintaining a balanced equilibrium. ${ }^{17}$

\section{Laboratory Evaluation}

The laboratory investigation profile of these patients must have plasma glucose, osmolality, creatinine, electrolytes with anion gap calculated, serum ketones, arterial blood gases, and complete blood count with differential. Investigations like blood urea nitrogen, urine analysis, and urine ketones are also useful. ${ }^{1,18}$ Whenever clinically indicated, electrocardiogram, chest X-ray, and urine, sputum, or blood cultures should also be done. ${ }^{18} \mathrm{HbA} 1 \mathrm{c}$ is useful to assess the chronology of diabetes control differentiating poorly controlled or undiagnosed diabetes from otherwise well-controlled patient. ${ }^{19}$ The serum glucose level is usually in the range of $600-1200 \mathrm{mg} /$ $\mathrm{dL}$. The higher level of glucose will lead to higher serum osmolarity and the higher degree of dehydration. Leukocytosis is common in HHS. ${ }^{10}$ The leukocytosis can be caused by higher level of cortisol and catecholamines associated with stress of hyperglycemia and dehydration. Whenever leukocytes count is greater than $25,000 / \mathrm{dL}$, thorough investigations to assess possibility of infection becomes mandatory. 2,20

The sodium level is falsely low. This pseudohyponatremia is because of the osmotic movement of water from the intracellular compartment to the extracellular compartment. ${ }^{2}$ The correct or true sodium level is calculated using the formula:

Corrected sodium $=$ Measured sodium $+[(($ Serum glucose -100$) / 100) \times 1.6]$

The potassium level might be high or low. The decreased level of insulin can lead to an extracellular shift of potassium. However, because of urinary potassium losses, the total body potassium is low. The bicarbonate level is usually close to normal in HSS, around 8-12 $\mathrm{mEq} / \mathrm{L}$, because production of ketones is nil or minimal. The anion gap in HSS is close to normal. The anion gap is determined by the formula: $(\mathrm{Na}+\mathrm{K})-(\mathrm{Cl}+\mathrm{HCO})$.

If the anion gap is high in HSS, it is usually because of the production of lactic acid from tissue hypoperfusion and decreased circulation. The magnesium level might be low in HSS.

In HSS, hyperphosphatemia may occur in presence of rhabdomyolysis. Administration of insulin and adequate hydration usually lower the phosphorus level by increasing phosphorous movement back into cells as well as increasing renal excretion as end-organ perfusion improves.

The serum osmolarity levels between 320 and $400 \mathrm{mOsm} / \mathrm{kg}$ is very common in HSS. Normal serum osmolarity is around $280-290$ $\mathrm{mOsm} / \mathrm{kg}$.

Ketonemia is very minimal in HSS. Arterial blood gases in HSS patient's shows the $\mathrm{pH}$ is usually above or around 7.30 while the $\mathrm{pCO}_{2}$ might be low from hyperventilation. Acidosis in HSS is mainly as a result of dehydration and compromised end-organ perfusion. The BUN and creatinine values are higher in most of the patients suggesting prerenal azotemia. The level of serum enzymes like creatinine kinase, aldolase, and transaminases are usually high from hemoconcentration and dehydration. Urine specific gravity is high in HSS. Glycosuria and ketonuria are also present.

\section{Treatment}

Treatment of HHS and DKA is similar. Correction of the fluid and electrolyte imbalance along with the administration of insulin are important components. Identification and treatment of any precipitating events should be done simultaneously.

The guidelines differ with regards to selection of fluid at higher sodium levels and timing for starting intravenous insulin.

\section{Correction of the Fluid}

Aggressive fluid therapy is the first step in management of $\mathrm{HHS}^{9}{ }^{9}$ The aim of fluid therapy is to restore $50 \%$ of the total deficit in the first 12 hours and improve renal perfusion. Patients with $\mathrm{HHS}$ often have a large fluid deficit around $100-200 \mathrm{~mL} / \mathrm{kg} .{ }^{21}$ The HHS patients suffer severe degree of sodium and chloride deficit. Sodium chloride $0.9 \%$ is the most preferred crystalloid. ${ }^{7,21,22}$ The goal of treatment is to achieve decrease in osmolality by 3-8 $\mathrm{mOsmol} / \mathrm{kg} / \mathrm{hour}$. It is critical to do serum osmolality (measured or calculated), blood glucose, and serum sodium measurements hourly in this period. ${ }^{1}$ Both guidelines recommend to start with $1 \mathrm{~L}$ of $0.9 \%$ saline as intravenous fluid. The isotonic fluid should be given at rate of $15-20 \mathrm{~mL} \mathrm{~kg} / \mathrm{hour}$.

Depending on the state of hydration and corrected serum sodium levels, tonicity and volume of subsequent crystalloid solutions are selected. ${ }^{7}$ One recent current diabetic review suggests that in the presence of an exclusive water shift, the quantitative method based on the comparison of measured plasma sodium and the plasma sodium expected can give better information of patient's true hydroelectrolytic status. This can help to select proper composition of fluid. They proposed a seven-step algorithm to compute an accurate estimate of water shift. ${ }^{21}$

As per the ADA guidelines, after the first hour of fluid replacement patients with a normal or high corrected sodium can be shifted to $0.45 \%$ sodium chloride solution. If the blood glucose decreases to less than $300 \mathrm{mg} / \mathrm{dL}$, dextrose $5 \%$ containing saline should be the hydrating fluid. ${ }^{23}$ Whenever the fall in plasma osmolality and glucose levels plateaus in the presence of sufficient positive fluid status, only in these situations UK guidelines recommend $0.45 \%$ sodium chloride solution. ${ }^{22}$

It is important to replace the urinary losses to achieve electrolytes and water deficit correction. ${ }^{7}$ During fluid therapy, the renal function and comorbidities like heart failure should always be taken into account.

\section{Correction of Electrolyte Abnormalities}

Ensuring adequate renal function prior to correction of potassium deficits is mandatory. The ADA recommends addition of 20-30 $\mathrm{mEq}$ potassium in every liter of intravenous fluid whenever serum potassium is $<5.2 \mathrm{mEq} / \mathrm{L} .{ }^{23,24}$ As per UK guidelines, in patients having good urine output with serum potassium value $<5.5 \mathrm{mEq} / \mathrm{L}, 40 \mathrm{mEq} / \mathrm{L}$ of potassium should be added per liter of normal saline. ${ }^{22}$ Intravenous insulin drives potassium in the intracellular compartment. It can worsen hypokalemia; to avoid this complication, it is recommended that whenever the serum potassium value is $<3 \mathrm{mEq} / \mathrm{L}$, it is essential to correct the potassium deficiency before starting insulin. ${ }^{2}$ Phosphate replacement should 
be done only after confirming phosphate deficiency. However, if hypophosphatemia persists after the acute phase, correction of phosphate is recommended by the UK guidelines. ${ }^{22}$

\section{Insulin}

There is difference of opinion with regards to optimal time to start insulin therapy. ${ }^{24}$ As per the ADA guidelines before starting intravenous insulin it is important to confirm starting of fluid therapy, correction of hypokalemia and fall in serum osmolality reaches plateau. Intravenous insulin is started at a 0.1 units/ $\mathrm{kg}$ bolus followed by 0.14 units $/ \mathrm{kg} / \mathrm{hour}$ or 0.1 units $/ \mathrm{kg} / \mathrm{hour}$. Whenever decrease in the glucose level is less than $50-70 \mathrm{mg} / \mathrm{dL} /$ hour, the insulin dose should be increased every hour to achieve steady glucose decline. If glucose falls to less than $300 \mathrm{mg} / \mathrm{dL}$, then insulin and dextrose infusion rate should be altered to achieve goal of glucose level between 250 and $300 \mathrm{mg} / \mathrm{dL}$. It is important to maintain blood glucose levels in this range until the serum osmolality and mental status is normalized. ${ }^{23}$

As per the UK guidelines, if the fall in glucose level is less than $90 \mathrm{mg} / \mathrm{dL} /$ hour, 3- $\beta$-hydroxybutyrate concentrations $>10.4 \mathrm{mg} /$ $\mathrm{dL}$ with adequate positive fluid status, then the intravenous insulin should be started. Starting dose of intravenous insulin is low $(0.05$ units $/ \mathrm{kg} / \mathrm{hour})$. During the initial 24 hours of treatment, fall in the glucose level should not be less than $180-270 \mathrm{mg} / \mathrm{dL}^{22}$

Risk of arterial as well as venous thromboembolism in these patients is similar to conditions like acute renal failure, sepsis, or acute events connective tissue disorders. ${ }^{25-27}$ The UK guidelines recommend that low-molecular-weight heparin should be given to every patient every day during hospital stay unless it is contraindicated. ${ }^{22}$

Cerebral edema is the most dreaded complication in both DKA and HSS. The risk is higher in pediatric patients. ${ }^{28}$ To avoid complications associated with rapid overcorrection, the ADA as well as UK guidelines recommend to monitor serum osmolality vigilantly. Decrease in plasma osmolarity and glucose levels in serum must be gradual. If the decline is too rapid and the brain is not able to eliminate idiogenic osmoles at the same rate as the decline in serum osmolarity, then the chances of fluid moving into the brain cell and causing swelling are higher. ${ }^{9}$ Maintaining the blood glucose level fall rate between 72 and $108 \mathrm{mg} /$ hour is recommended to be safe and the plasma sodium level should not decrease more than $10 \mathrm{mEq} / \mathrm{L}$ in 24 hours. $^{22,23,29}$ Hypertonic saline solutions, infusion of mannitol, and mechanical ventilation are also suggested while treating cerebral edema. ${ }^{23}$

The HHS is considered to be resolved when the patient has recovered mental alertness, serum osmolality levels decreases to $<310 \mathrm{mOsmol} / \mathrm{kg}$, and the glucose level is less than $250 \mathrm{mg} / \mathrm{dL}^{2,23}$

\section{Mortality}

Higher age at presentation and coexisting comorbidities add to the higher mortality. ${ }^{11}$ Mortality rates varies from 5 to $35 \% .{ }^{7}$ Most important predictors are age and the degree of hyperosmolarity. With age $>65$ years and serum osmolarity $>375 \mathrm{mOsmol} / \mathrm{L}$, the risk of fatal outcome is greatest. ${ }^{2}$

Strategies to avoid readmissions, mortality, and longterm morbidity are equally important. As apart from medical comorbidities, the other reasons that contribute to development of HHS are substance- or alcohol-use disorders, insulin nonadherence, and psychiatric disorders. Training of treating doctors, patients, and their families with regards to early detection of acute decompensated diabetes and strategies to prevent and/or treat would be beneficial. ${ }^{24}$

\section{References}

1. Elizabeth Kerr D, Wenham T, Munir A. Endocrine problems in the critically ill 1: diabetes and glycaemic control. BJA Educ 2017;17(11):370-376. DOI: 10.1093/bjaed/mkx024.

2. Fayfman M, Pasquel FJ, Umpierrez GE. Management of hyperglycemic crises. Med Clin North Am 2017;101(3):587-606. DOI: 10.1016/j. mcna.2016.12.011.

3. Pasquel FJ, Umpierrez GE. Hyperosmolar hyperglycemic state: A historic review of the clinical presentation. Diagnosis, and Treatment 2014;37(11):3124-3131. DOI: 10.2337/dc14-0984.

4. Badawi O, Waite MD, Fuhrman SA, et al. Association between intensive care unit-acquired dysglycemia and in-hospital mortality. Crit Care Med 2012;40(12):3180-3188. DOI: 10.1097/CCM.0b013e3182656ae5.

5. Rosenbloom AL. Hyperglycemic hyperosmolar state: an emerging pediatric problem. J Pediatr 2010;156(2):180-184. DOI: 10.1016/j. jpeds.2009.11.057.

6. McDonnell CM, Pedreira CC, Vadamalayan B, et al. Diabetic ketoacidosis, hyperosmolarity and hypernatremia: are highcarbohydrate drinks worsening initial presentation? Pediatr Diabetes 2005;6(2):90-94. DOI: 10.1111/j.1399-543X.2005.00107.x.

7. Kitabchi AE, Umpierrez GE, Murphy MB. Chapter 54 diabetic ketoacidosis and hyperosmolar state. 2015. 799-814.

8. Gershkovich B, English SW, Doyle M, et al. Choice of crystalloid fluid in the treatment of hyperglycemic emergencies: a systematic review protocol. 2019(1):1-7. DOI: 10.1186/s13643-019-1130-5.

9. Adeyinka A, Kondamudi NP, Brooklyn T, Hyperosmolar Hyperglycemic Nonketotic Coma (HHNC, Hyperosmolar Hyperglycemic Nonketotic Syndrome). 2019.

10. Ennis ED, Stahl EJVBKR. The hyperosmolar hyperglycemic syndrome. Diabetes Rev 1994;2:115-126.

11. Wachtel TJ, Silliman RA, Lamberton P. Prognostic factors in the diabetic hyperosmolar state. J Am Geriatr Soc 1987;35(8):737-741. DOI: 10.1111/j.1532-5415.1987.tb06351.x.

12. Care D, Suppl SS. Classification and diagnosis of diabetes: Standards of medical care in diabetes 2019. Diabetes Care 2019;42(January):S13S28.

13. Gerich JE, Martin MMRL. Clinical and metabolic characteristics of hyperosmolar nonketotic coma. Diabetes 1971;20(4):228-238. DOI: 10.2337/diab.20.4.228.

14. Dhatariya KK, Vellanki P, Treatment of Diabetic Ketoacidosis ( DKA)/ Hyperglycemic Hyperosmolar State ( HHS): Novel Advances in the Management of Hyperglycemic Crises ( UK Versus USA). 2017.

15. Wachtel TJ, Silliman RALP. Predisposing factors for the diabetic hyperosmolar state. Arch Intern Med 1987;147(3):499-501. DOI: 10.1001/archinte.1987.00370030103020.

16. Chupin M, Charbonnel BCF. C-peptide blood levels in keto-acidosis and in hyperosmolar non-ketotic diabetic coma. Acta Diabetol Lat 1981;18(2):123-128. DOI: 10.1007/BF02098997.

17. Nugent BW. Hyperosmolar hyperglycemic state. Emerg Med Clin North Am 2005;23(3):629-648. DOI: 10.1016/j.emc.2005.03.006.

18. Maletkovic JDA. Diabetic ketoacidosis and hyperglycemic hyperosmolar state. Endocrinol Metab Clin North Am 2013;42(4):677695. DOI: 10.1016/j.ecl.2013.07.001.

19. Np K. Hyperosmolar Hyperglycemic nonketotic coma (HHNC, hyperosmolar Hyperglycemic Nonketotic syndrome). 2019. 1-7.

20. Slovis CM, Mork VG, Slovis RJBR. Diabetic ketoacidosis and infection: leukocyte count and differential as early predictors of serious infection. Am J Emerg Med 1987;5(1):1-5. DOI: 10.1016/07356757(87)90280-4.

21. Baldrighi M, Sainaghi PP, Bellan M, et al. Hyperglycemic hyperosmolar state: A pragmatic approach to properly manage sodium derangements. Curr Diabetes Rev 2018;14(6):534-541. DOI: 10.2174/ 1573399814666180320091451. 
22. Scott AR. Joint British Diabetes Societies (JBDS) for inpatient care JBDS hyperosmolar hyperglycemic guidelines group. management of hyperosmolar hyperglycaemic state in adults with diabetes. Diabet Med 2015;32(6):714-724. DOI: 10.1111/dme. 12757.

23. Kitabchi AE, Umpierrez GE, Miles JM, et al. Hyperglycemic crises in adult patients with diabetes. Diabetes Care 2009;32(7):1335-1343. DOI: 10.2337/dc09-9032.

24. French EK, Donihi AC, Korytkowski MT, State of the Art Review Diabetic ketoacidosis and hyperosmolar hyperglycemic syndrome: review of acute decompensated diabetes in adult patients. 2019.
25. Whelton MJ, Walde DHC. Hyperosmolar non-ketotic diabetes coma - with particular reference to vascular complications. BMJ 1971;1(5740):85-86. DOI: 10.1136/bmj.1.5740.85.

26. Keenan CR, Murin SWR. High risk for venous thrombo- embolism in diabetics with hyperosmolar state: comparison with other acute medical illnesses. J Thromb Haemost 2007;5(6):1185-1190. DOI: 10.1111/j.1538-7836.2007.02553.x.

27. Paton RC. Haemostatic changes in diabetic coma. Diabetologia 1981;21(3):172-177. DOI: 10.1007/BF00252650.

28. Statements ADA. Hyperglycemic Crises in Adult Patients 2009;32(7).

29. Adrogue HJ, Hypernatremia MN. N Engl J Med 2000;342(20): 1493-1499. DOI: 10.1056/NEJM200005183422006. 